\title{
Square-Root Algorithms for Least-Squares Estimation
}

MARTIN MORF, MEMBER, IEEE, AND THOMAS KAILATH, FELLOW, IEEE

\begin{abstract}
We present several new algorithms, and more generally a new approach, to recursive estimation algorithms for linear dynamical systems. Earlier results in this area have been obtained by several others, especially Potter, Golub, Dyer and McReynolds, Kaminski, Schmidt, Bryson, and Bierman on what are known as square-root algorithms. Our results are more comprehensive. They also show how constancy of parameters can be exploited to reduce the number of computations and to obtain new forms of the Chandrasekhar-type equations for computing the filter gain. Our approach is essentially based on certain simple geometric interpretations of the overall estimation problem. One of our goals is to attract attention to non-Riccati-based studies of estimation problems.
\end{abstract}

\section{INTRODUCTION}

$\mathrm{W}$ E SHALL present some new algorithms, and more generally a new approach, for linear least-squares estimation in the discrete-time state-space system,

$$
\begin{aligned}
x_{i+1} & =\Phi_{i} x_{i}+\Gamma_{i} u_{i} \\
y_{i} & =H_{i} x_{i}+v_{i}, \quad i \geqslant 0 \\
E v_{i} v_{j}^{\prime} & =R_{i} \delta_{i j}, E v_{i} u_{j}^{\prime}=C_{i} \delta_{i j}, E u_{i} u_{j}^{\prime}=Q_{i} \delta_{i j} \\
E x_{0} x_{0}^{\prime} & =\Pi_{0}, E u_{i} x_{0}^{\prime}=0=E v_{i} x_{0}^{\prime} .
\end{aligned}
$$

The matrices $\{\Phi, \Gamma, H, Q, C, R\}$ are assumed to be known and all the random variables are assumed to have zero mean. Primes will denote transposes. The dimensions of the vectors $u, x, y$ are $m, n, p$, respectively.

It is by now well known (beginning with [1]) that with this system we can associate a so-called matrix Riccatitype difference equation (recursion),

$$
P_{i+1}=\Phi_{i} P_{i} \Phi_{i}^{\prime}+\Gamma_{i} Q_{i} \Gamma_{i}^{\prime}-\tilde{K}_{i} \tilde{K}_{i}^{\prime}, \quad P_{0}=\Pi_{0}
$$

where

$$
\begin{aligned}
\tilde{K}_{i} & =K_{i}\left(R_{i}^{\epsilon}\right)^{-\frac{T}{2}} \\
K_{i} & =\Phi_{i} p_{i} H_{i}^{\prime}+\Gamma_{i} C_{i}^{\prime} \\
R_{i}^{\epsilon} & =R_{i}+H_{i} P_{i} H_{i}^{\prime}>0
\end{aligned}
$$

Manuscript received August 12, 1974; revised February 24, 1975. Paper recommended by $\mathbf{H}$. W. Sorenson, Chairman of the IEEE S-CS Estimation and Identification Committee. This work was supported in part by the Air Force Office of Scientific Research, Air Force Systems, under Contract AF44-620-74-C-0068, in part by the Joint Services Electronics Program under Contract N00014-67-A-0112-0044, and in part by a fellowship of the Hasler Foundation, Berne, Switzerland.

The authors are with the Information Systems Laboratory and the Department of Electrical Engineering, Stanford University, Stanford, Calif. 94305 . and

$$
\begin{aligned}
P_{i}= & \text { the variance of the error } \\
& \text { in the one step prediction of } x_{i} \\
& =E\left[x_{i}-\hat{x}_{i \mid i-1}\right]\left[x_{i}-\hat{x}_{i \mid i-1}\right]^{\prime} .
\end{aligned}
$$

Let

$\hat{x}_{i \mid i-1}=$ the linear least-squares estimate of $x_{i}$ given

$$
y[0, i-1]
$$

where we use the notation

$$
\left[y_{j}^{\prime}, \cdots, y_{i}^{\prime}\right]^{\prime} \triangleq y[j, i] .
$$

Now we can write the estimator equation

$$
\hat{x}_{i+1 \mid i}=\Phi_{i} \hat{x}_{i \mid i-1}+\tilde{K}_{i} p_{i}, \quad \hat{x}_{0 \mid-1}=0
$$

where

$$
\nu_{i}=\left(R_{i}\right)^{-\frac{1}{2}}\left(y_{i}-H_{i} \hat{x}_{i \mid i-1}\right)=\text { the normalized innovations in } y_{i}
$$

$$
\text { given } y[0, i-1] \text {. }
$$

and we have used the notation

$$
\begin{aligned}
& R^{\frac{1}{2}}=\text { any matrix such that } R^{\frac{1}{2}} R^{\frac{T}{2}}=R \\
& R^{\frac{T}{2}}=\left(R^{\frac{1}{2}}\right)^{\prime} .
\end{aligned}
$$

The above algorithm, known as the Kalman filter, has dominated the estimation field since the appearance in 1960 of the fundamental paper [1]. In particular, the Riccati equation has been fairly exhaustively studied (see [3] for a recent survey), and the Kalman filter has been widely used. Despite this remarkable success it is useful to develop alternative algorithms. The most important reason, perhaps, is that diversity is valuable for its own sake, provided there is a fair possibility that the new algorithms have potential advantages in some respects, among which we should include things like the complexity, accuracy, and the conditioning of computations.

In the last few years, two sets of new algorithms have been developed. One is the family of "square-root" algorithms associated with the names of Potter [4], Golub [5], Hanson and Lawson [6], Dyer and McReynolds [7], Schmidt [8], Kaminski and Bryson [9], [10], and Bierman [11], [12]. These algorithms recursively compute square 
roots of the variance matrix (or its inverse), thus ensuring that the square is always nonnegative definite and also allowing more significant digits to be used in the actual calculation (the "condition number" for $P_{i}$ is the square of that for $P^{\frac{1}{2}}$ ). However, since $P^{\frac{1}{2}}$ is also $n \times n$, the price generally paid in such algorithms is a larger number of computations.

More recently, we have been studying [13]-[17] another class of algorithms for constant (time-invariant) systems in which the Riccati-type difference equation is replaced by another set of equations, which we have named as being of Chandrasekhar-type. The main feature of these new algorithms is that they work with square-roots of $\delta P_{i}$ rather than of $P_{i}$, the point being that for constant systems the rank of $\delta P_{i}$ is often much less than that of $P_{i}$, so that the number of quantities to be determined can often be reduced from $0\left(n^{2}\right)$ to $O(n)$. In addition we have the same advantages on condition number as the square-root algorithms, and while nonnegative-definiteness of $P_{i}$ $=\Sigma_{i} \delta P_{i}$ cannot be guaranteed, the algorithms are potentially better behaved in this regard than is direct solution of the Riccati equation. While the new algorithms based on the Chandrasekhar-type equations have several other interesting features [for example, the problem of degenerate directions extensively studied by Bucy et al. [18], [19] can be handled without difficulty and also the equations have many connections with earlier work by Levinson, Durbin, Whittle, Wiggins and Robinson, and others (see references in [16])], our aim in this paper is to explore the connections between the new algorithms and the earlier square-root algorithms.

Section II gives a quick look at some of our results and methods, applied for simplicity to models with $E v_{i} u_{j}^{\prime}$ $=C_{i} \delta_{i j}=0$. We first present an "instantaneous" derivation of a form of the previously known (covariance) squareroot array-algorithms. Then we show how the assumption of constant model parameters can be exploited to reduce the number of variables in the array algorithm. Finally by explicitly specifying the orthogonal transformations used in the array methods we obtain explicit updating equations that give "fast" forms of the Potter-Andrews squareroot equations. The remaining sections treat several generalizations and new forms. The results so far will have been derived by starting with an initial matrix, which is then factored in different ways to give the square-root arrays; however, the initial matrix is "pulled out of the air." When in Section III, we begin to study the general model $\left(C_{i} \neq 0\right)$ and to ask for smoothing results and information forms, we begin to see the need for some general underlying principles. This is provided in Section IV via a simple geometric picture that allows us to write down all the arrays as (cross-correlation) coefficient matrices for representing certain variables of interest in terms of other given (sequentially orthonormalized) variables.

Section $V$ shows that square-root information algorithms can be derived from the covariance forms and vice versa by a simple inversion of triangular matrices.
This leads to a new duality between the covariance and information forms, which is also capable of handling the case $C_{i} \neq 0$ not readily obtained via the standard duality argument [9], [10]. In Section VI we give the appropriate arrays for problems where covariance information is given in place of a state model.

For reasons of space, we put off discussion of smoothing problems to another paper, but in Appendix A we give as an example a new and potentially simpler fixed-point smoothing formula provided by our approach. Appendix $B$ indicates how our results can be extended to certain classes of time-variant systems and Appendix $\mathrm{C}$ provides some results for continuous-time and continuous-time discrete measurement systems.

Finally we may emphasize that the numerical promise of the various algorithms proposed here remains to be carefully studied. However, it is important that we have a new family of equations and methods that provide alternatives to the almost total emphasis in the last decade on the Riccati-equation Kalman filter algorithms.

The results of Section II were first presented at the 1973 IEEE Decision and Control Conference, San Diego, California, in December 1973, [29]. An earlier version of this paper appeared as [22]; see also Section VII for other historical remarks and acknowledgments, especially [23].

\section{Some SQlare-Root Algorithms}

To bring out the main ideas more clearly in this section, we make the simplifying assumption that $E v_{i} u_{j}^{\prime}=0$.

Now consider the following (nonnegative-definite) matrix, which arises also in the positive-real lemma, quadratic control, etc.,

$$
\mathfrak{K}=\left[\begin{array}{ll}
\multicolumn{1}{c}{P} & \multicolumn{1}{c}{n} \\
R_{i}+H_{i} P_{i} H_{i}^{\prime} & H_{i} P_{i} \Phi_{i}^{\prime} \\
\Phi_{i} P_{i} H_{i}^{\prime} & \Phi_{i} P_{i} \Phi_{i}^{\prime}+\Gamma_{0} Q_{i} \Gamma_{i}^{\prime}
\end{array}\right],
$$

We now claim that it is clear by inspection that we can factor the $2 \times 2$ block matrix $\mathfrak{R}$ as

$$
\mathscr{N}=\mathcal{e}_{1} e_{1}^{r}, \quad \text { where } \mathcal{e}_{1}={ }_{n}\left[\begin{array}{ccc}
p & n & m \\
R_{i}^{\frac{1}{2}} & H_{i} P_{i}^{\frac{1}{2}} & 0 \\
0 & \Phi_{i} P_{i}^{\frac{1}{2}} & \Gamma_{i} Q_{i}^{\frac{1}{2}} \\
p & n & m
\end{array}\right] .
$$

Next we note that the $(2,2)$ element of the block matrix $\mathscr{N}$ is the variance of the random variable

$$
x_{i+1}-\hat{x}_{i+1 \mid i-1}=\Phi_{i} \tilde{x}_{i \mid i-1}+\Gamma_{i} u_{i} .
$$

By using (1) and (8), we can rewrite this variable as

$$
\Phi_{i} \tilde{x}_{i \mid i-1}+\Gamma_{i} u_{i}=\tilde{x}_{i+1 \mid i}+\tilde{K}_{i} \nu_{i}
$$

from which, by comparing variances, we get

$$
\Phi_{i} P_{i} \Phi_{i}^{\prime}+\Gamma_{i} Q_{i} \Gamma_{i}^{\prime}=P_{i+1}+\tilde{K}_{i} \tilde{K}_{i}^{\prime}, \quad \tilde{K}_{i}=K_{i}\left(R_{i}^{\epsilon}\right)^{-\frac{T}{2}},
$$


an identity that may also be obtained from the Riccati equation (2). Now using (13) and the definitions (3)-(5), we can rewrite the matrix $\Re$ of $(10)$ as

$$
\mathfrak{N}=\left[\begin{array}{cc}
R_{i}^{\epsilon} & K_{i}^{\prime} \\
K_{i} & P_{i+1}+K_{i}\left(R_{i}^{\epsilon}\right)^{-1} K_{i}^{\prime}
\end{array}\right]
$$

which can again be factored by inspection as

$$
\Re=e_{2} \bigodot_{2}^{\prime}, \quad e_{2}=\left[\begin{array}{ccc}
p & n & m \\
\left(R_{i}^{\epsilon}\right)^{\frac{1}{2}} & 0 & 0 \\
\tilde{K}_{i} & p_{i+1}^{\frac{1}{2}} & 0
\end{array}\right] p .
$$

But since $\mathcal{C}_{1}$ and $\mathcal{C}_{2}$ are both factors of $\Re$ they have to be related by an orthogonal transformation $\mathscr{T}\left(\mathscr{T}^{\prime} \mathcal{T}^{\prime}=I\right)$. We therefore see that a technique for updating a "prearray" is to apply to $\mathcal{C}_{1}$ any orthogonal transformation 9 such that we force a pattern of zeros as shown in (16), where $\mathscr{X}_{1}$ is a basis of the $p$-column vectors of the first block row of $e_{1}$ and $X_{3}$ forms a basis for columns of $e_{1}$ with zeros in the first $p$-components.

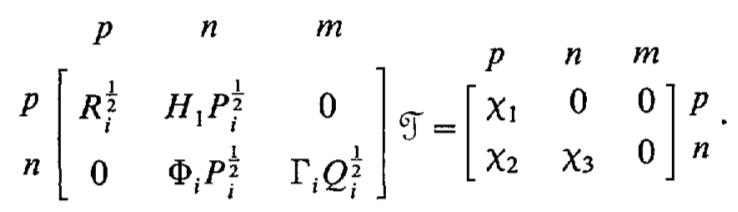

Then the quantities marked $\left\{\mathscr{X}_{i}\right\}$ will have to be equal (apart from orthogonal transformations) to $\left(R_{i}^{\epsilon}\right)^{\frac{1}{2}}, \tilde{K}_{i}$, and $P_{i+1}^{\frac{1}{2}}$, respectively. (The nonuniqueness is irrelevant to the final calculation of $P_{i+1}$ [or $\hat{x}_{i+1 \mid i}$ via (8)] and therefore, in later discussions we shall disregard it.)

The reader can now verify that what we have in (16) is the known square-root algorithm, except that the separately treated "time-update" and "measurementupdate" steps [9], [10] have been combined into one. We may note that our derivation is almost "instantaneous," especially as compared with those in the literature. For reference, we also give the time update and measurementupdate formulas. In the measurement up-date, we compute the "filtered" error variance $P^{\frac{1}{2}}$ i| from knowledge of the "predicted" error variance $P^{\frac{1}{2}} \triangleq P_{i \mid i-1}^{\frac{1}{2}}$, via the arrays

$$
\left[\begin{array}{ll}
R_{i}^{\frac{1}{2}} & H_{i} P_{i}^{\frac{1}{2}} \\
0 & p_{i}^{\frac{1}{2}}
\end{array}\right] \mathscr{T}_{1}=\left[\begin{array}{ll}
\left(R_{i}^{\epsilon}\right)^{\frac{1}{2}} & 0 \\
\tilde{K}_{i} & p_{i \mid i}^{\frac{1}{2}}
\end{array}\right]
$$

The filtered state estimate is given by $\hat{x}_{i \mid i}=\hat{x}_{i \mid i-1}+\tilde{K}_{i v i}$ and $\nu_{i}$ by (9). In the time-update, we compute $P_{i+1}^{\frac{1}{2}}$ from knowledge of $P_{i}^{\frac{1}{2}}$ via

$$
\left[\begin{array}{ll}
\Phi_{i} P_{i}^{\frac{1}{2} n} & \Gamma_{i} Q_{i}^{\frac{1}{2}}
\end{array}\right] \Im_{2}=\left[\begin{array}{ll}
P_{i+1}^{\frac{1}{2}} & 0
\end{array}\right]
$$

and the predicted state estimate from $\hat{x}_{i+1 \mid i}=\Phi_{i} \hat{x}_{i \mid j}$. The formulas (17), (18) can be verified by squaring up both sides. We also note that a little algebra will show how (17) and (18) can be merged into our formula (16). The formula (18) was first given by Schmidt [8], while the form (17) was first given by Kaminski [9], [10] (who obtained it by applying a certain duality, further discussed in Section $\mathrm{V}$, to a procedure of Dyer and McReynolds [7]). Actually the first square-root formulas were given by Potter (see [4], [4a]), whose formulas were actually somewhat more explicit since they effectively specified $\sigma_{1}$. This will be explored below, but let us first explain how the size of the arrays involved in the square-root algorithms can often be substantially reduced from $0\left(n^{2}\right)$ to $0(n)$ in certain problems with time-invariant parameters $(F, G, H, Q, R)$.

\section{Fast Algorithms for Time-Invariant Systems}

When the model parameters are constant, as noted earlier [13]-[17], computational benefits can be obtained by working with square roots not of $P_{i}$ but of $\delta P_{i}$. We now explore this point here.

To introduce the main ideas, let us first consider the special (but prototype) case of known initial conditions, so that

$$
\Pi_{0}=0 \text {. }
$$

In this case, we have shown previously [13] that $\delta P_{i}$ is nonnegative-definite and of nonincreasing rank,

$$
\delta P_{i}=P_{i+1}-P_{i} \geqslant 0, \quad \operatorname{rank} \delta P_{i} \geqslant \operatorname{rank} \delta P_{i+1} .
$$

Therefore, we can factor $\delta P_{i}$ as

$$
\delta P_{i}=\tilde{L}_{i} \tilde{L_{i}^{\prime}}, \quad \tilde{L_{i}}=n \times \alpha
$$

where

$$
\alpha=\operatorname{rank} \text { of } \delta P_{i}=P_{1}-\Pi_{0}=\Gamma Q \Gamma^{\prime}
$$

We note that

$\alpha \leqslant m, \quad$ the number of inputs in the model (1).

(For reference, we may note that in [14] we factored $\delta P_{i}$ as $Y_{i} M_{i} Y_{i}^{\prime}$, and obtained separate recursions for $Y_{i}$ and $M_{i}$; here it is more convenient to work with $\tilde{L}_{i}=Y_{i} M_{i}^{\frac{1}{2}}$.) We shall now show that the arrays in (16) can be replaced by

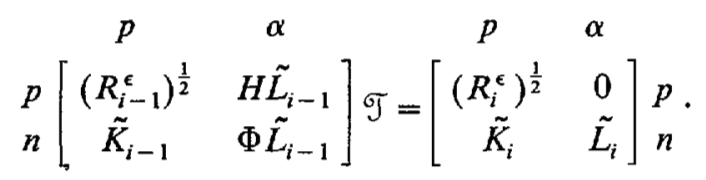

Since $\alpha \leqslant m$ and $m$ can often be less than $n$, the use of (24) may result in a substantial reduction in storage and computational requirements compared to (16). 
Derivation of the Fast Algorithm for $\Pi_{0}=0$

If $P_{i+1}=P_{i}+\tilde{L}_{i} \tilde{L}_{i}^{\prime}$ we can write, for some orthogonal matrix $\sigma_{0}$,

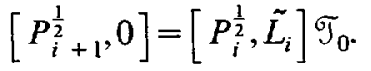

Therefore, we can write $C_{1}$ of (11) as follows (where by $A \sim B$ we shall mean $A A^{\prime}=B B^{\prime}$ or $A_{0} \mathscr{T}=B_{0}$ with $\mathcal{T}^{\prime} \mathcal{T}^{\prime}$ $=I$, and $A_{0}=[A, 0]$ and $B_{0}=[B, 0]$ are matrices suitably expanded by zero columns to the same dimensions):

$$
\begin{aligned}
& \mathcal{C}_{1} \sim\left[\begin{array}{lll}
R^{\frac{1}{2}} & H P_{i}^{\frac{1}{2}} & 0 \\
0 & \Phi P_{i}^{\frac{1}{2}} & \Gamma Q^{\frac{1}{2}}
\end{array}\right] \\
& \sim\left[\begin{array}{llll}
R^{\frac{1}{2}} & H P_{i-1}^{\frac{1}{2}} & H \tilde{L}_{i-1} & 0 \\
0 & \Phi P_{i-1}^{\frac{1}{2}} & \Phi{\tilde{L_{i-1}}} & \Gamma Q^{\frac{1}{2}}
\end{array}\right] \\
& \sim\left[\begin{array}{ccc}
\left(R_{i-1}^{\epsilon}\right)^{\frac{1}{2}} & 0 & H \tilde{L}_{i-1} \\
\tilde{K}_{i-1} & P_{i}^{\frac{1}{2}} & \Phi \tilde{L}_{i-1}
\end{array}\right]
\end{aligned}
$$

where the last array is obtained by applying (16) to the first-, second-, and fourth-block columns. We can also write the $e_{2}$ of (15) as

$$
\mathcal{C}_{2} \sim\left[\begin{array}{cll}
\left(R_{i}^{\epsilon}\right)^{\frac{1}{2}} & 0 & 0 \\
\tilde{K}_{i} & P_{i+1}^{\frac{1}{2}} & 0
\end{array}\right] \sim\left[\begin{array}{ccc}
\left(R_{i}^{\epsilon}\right)^{\frac{1}{2}} & 0 & 0 \\
\tilde{K}_{i} & P_{i}^{\frac{1}{2}} & \tilde{L}_{i}
\end{array}\right] .
$$

But $e_{1}$ and $e_{2}$ are equivalent modulo orthogonal transformations on the right, so that ignoring the (block) column with $P_{i}^{\frac{1}{2}}$, we get the arrays given in (24).

The special choice $\Pi_{0}=0$ arises whenever the initial conditions are known or when they are deliberately set equal to zero in order to improve the computational accuracy by keeping the contribution of the quadratic terms in the Riccati equation low (see [25] and the end of Appendix $C$ ). The case $\Pi_{0}=0$ also arises in the dual quadratic regulator problems with zero terminal cost (see, e.g., [27]).

Another important special case is that corresponding to stationary signal models, as are often assumed in communication and system identification problems. Stationary signal processes are obtained by assuming the following.

1) $\Phi$ is stable (i.e., all its eigenvalues have magnitude less than unity).

2) $\Pi_{0}=\bar{\Pi}$, where $\bar{\Pi}$ is the unique nonnegativedefinite solution of the Lyapunov equation

$$
\bar{\Pi}=\Phi \bar{\Pi} \Phi^{\prime}+\Gamma Q \Gamma^{\prime} .
$$

With this special initial condition we have shown that [14]

$$
\delta P_{i}=P_{i+1}-P_{i} \leqslant 0, \quad \operatorname{rank} \delta P_{i} \geqslant \operatorname{rank} \delta P_{i+1} .
$$

Therefore, in this case we can factor $\delta P_{i}$ as

$$
\delta P_{i}=-\tilde{L}_{i} \tilde{L}_{i}^{\prime}, \quad \tilde{L_{i}}=n \times \alpha
$$

where

$\alpha=$ rank of $\delta P_{1}=\dot{P}_{1}-P_{0}=-\Phi \bar{\Pi} H^{\prime}\left(H \bar{\Pi} H^{\prime}+R\right)^{-1} H \bar{\Pi} \Phi^{\prime}$.

We note that $\alpha \leqslant p$, the number of outputs in the model (1). As far as the rank $\alpha$ goes, we note that, as pointed out in [14], it may be useful to reinitialize the problem after $n$ steps (where $n$ is the number of states), because by then any degenerate (constant) directions of $P_{i}$ will have been achieved. Now the appropriate arrays are [cf. (24)]

$$
p\left[\begin{array}{ll}
\multicolumn{1}{c}{p} & \alpha \\
\left(R_{i-1}^{\epsilon}\right)^{\frac{1}{2}} & \sqrt{-1} H \tilde{L}_{i-1} \\
K_{i-1} & \sqrt{-1} \Phi \tilde{L_{i-1}}
\end{array}\right] \circlearrowleft=\left[\begin{array}{ll}
\left(R_{i}^{\epsilon}\right)^{\frac{1}{2}} & 0 \\
\tilde{K}_{i} & \sqrt{-1} \tilde{L}_{i}
\end{array}\right]
$$

It might now seem that we will need complex arithmetic to transform the left-hand array to the one on the right. However note that the columns of $\left(R_{i}^{\epsilon}\right)^{\frac{1}{2}}$, i.e., of the first block row on the right hand form a set of real basis vectors for the space spanned by the columns of $\left\{\left(R_{i-1}^{\epsilon}\right)^{\frac{1}{2}}\right.$, $\left.\sqrt{-1} H \tilde{L_{i-1}}\right\}$. Therefore, $\overline{9}$ can be constructed without complex arithmetic, for instance by adding simple logic to the Householder algorithm (see below), which changes the sign in operations with imaginary elements [10, p. 77].

The same remarks apply to the general case of arbitrary $\Pi_{0}$ where $\delta P_{i}$ is indefinite and must therefore be factored in the form

$$
\begin{aligned}
\delta P_{i} & =\tilde{L}_{1 i} \tilde{L}_{1 i}^{\prime}-\tilde{L}_{2 i} \tilde{L}_{2 i}^{\prime} \\
& =\tilde{L}_{i} S \tilde{L_{i}^{\prime}}
\end{aligned}
$$

where $S$ is the signature matrix of $\delta P_{i}$. We forebear from giving the details. However, we may note that efficient numerical methods of determining the factorization (27) are studied in [28]. In particular. we emphasize that it is not necessary to determine the eigenvalues in order to find the signature matrix $S$.

Remark: We may note that it is the constancy of $H$ and $\Phi$ that allows us to carry out the above reductions [cf. (25)-(27)]. This suggests that we can relax our assumption of a constant (time-invariant) model to allow the parameters $R_{i}$ and $\Gamma_{i}, Q_{i}$ to be time-variant. This can in fact be done, as outlined in Appendix B.

\section{Explicit Updating Equations}

So far we have not needed to consider the explicit forms of the orthogonal transformations that update our arrays and, in fact, they can be quite arbitrary. However, a popular choice is to use the so-called Householder transformations, which have good numerical properties. Other choices are the Givens transformations and the modified 
Gram-Schmidt (MGS) method [2]-[5]. Bierman [11] has made the interesting observation that in the scalar measurement case $(p=1)$, use of an explicit form of the Householder transformation for $\mathscr{T}$ yields explicit measurement-update equations that contain the Potter squareroot equations. Namely, in the scalar time-invariant model form of (17),

$$
\left[\begin{array}{cc}
r_{i}^{\frac{1}{2}} & h_{i} P_{i}^{\frac{1}{2}} \\
0 & P_{i}^{\frac{1}{2}}
\end{array}\right] \tilde{\sigma}=\left[\begin{array}{cc}
\mp\left(r_{i}^{\epsilon}\right)^{\frac{1}{2}} & 0 \\
\tilde{k}_{i} & P_{i \mid i}^{\frac{1}{2}}
\end{array}\right]
$$

we can specify $\mathscr{T}$ explicitly as

$$
\begin{aligned}
\mathcal{T} & =I-U \beta U^{\prime}, \quad \beta=2\left(U^{\prime} U\right)^{-1} \\
U^{\prime} & =\left[\left(r_{i}^{\epsilon}\right)^{\frac{1}{2}} \pm\left(r_{i}\right)^{\frac{1}{2}}, \pm h_{i} P_{i}^{\frac{1}{2}}\right], \quad r_{i}^{\epsilon}=r_{i}+h_{i} P_{i} h_{i}^{\prime} .
\end{aligned}
$$

For the vector measurements case $(p>1)$, it can be shown [17] that a suitable $\mathcal{T}$ (equivalent to a product of $p$ scalar Householder transformations) can be written as

$$
\widetilde{J}=I-U\left\{\left[U^{\prime} U\right]_{+}\right\}^{-1} U^{\prime}
$$

where (with $I_{ \pm}$an arbitrary signature matrix)

$$
\begin{gathered}
{\left[R_{i}^{\frac{1}{2}}, H_{i} P_{i}^{\frac{1}{2}}\right] \Im_{0}=\left[-\left(R_{i}^{\epsilon}\right)^{\frac{1}{2}} I_{ \pm}, 0\right]} \\
U^{\prime}=I_{ \pm}\left(R_{i}^{\epsilon}\right)^{-\frac{1}{2}}\left[\left(R_{i}^{\epsilon}\right)^{\frac{1}{2}} I_{ \pm}+\left(R_{i}\right)^{\frac{1}{2}}, H_{i} P_{i}^{\frac{1}{2}}\right] \\
=\left[I+I_{ \pm}\left(R_{i}^{\epsilon}\right)^{-\frac{1}{2}}\left(R_{i}\right)^{\frac{1}{2}}, I_{ \pm}\left(R_{i}^{\epsilon}\right)^{-\frac{1}{2}} H_{i} P_{i}^{\frac{1}{2}}\right] \triangleq\left[U_{1}^{\prime}, U_{2}^{\prime}\right]
\end{gathered}
$$

and for this choice of $U^{\prime}$ we can write $\sigma=I-U U_{1}^{-T} U^{\prime}$ since

$$
\begin{array}{r}
{\left[U^{\prime} U\right]_{+}=\left[I+\left(R_{i}\right)^{\frac{T}{2}}\left(R_{i}^{\epsilon}\right)^{-\frac{T}{2}} I_{ \pm}+I_{ \pm}\left(R_{i}^{\epsilon}\right)^{-\frac{1}{2}}\left(R_{i}\right)^{\frac{1}{2}}+I\right]_{+}} \\
=I+I_{ \pm}\left(R_{i}^{\epsilon}\right)^{-\frac{1}{2}}\left(R_{i}\right)^{\frac{1}{2}}=U_{1}^{\prime},
\end{array}
$$

where we used the fact that for $L$ a lower triangular matrix $L=\left[L+L^{\prime}\right]_{+}$. [ $]_{+}$is the "lower-triangular-part" operator (if $M^{\frac{1}{2}}$ is lower triangular) defined such that $M_{+}+M_{-}=M$, where the

$$
i j \text { th element of } M_{+}=\left\{\begin{array}{ll}
0, & i<j \\
m_{i i} / 2, & i=j \\
m_{i j}, & i>j
\end{array}\right\} \text { and } M_{-}=\left(M_{+}\right)^{\prime} \text {. }
$$

The proof of (28) can be found by verification that

$$
\mathcal{T}^{\prime} \mathcal{T}^{\prime}=I-U\left\{\left[U^{\prime} U\right]_{+}\right\}^{-1}\left[\left[U^{\prime} U\right]_{+}+\left[U^{\prime} U\right]_{-}\right.
$$

$$
\left.-\left[U^{\prime} U\right]\right]\left\{\left[U^{\prime} U\right]_{-}\right\}^{-1} U^{\prime}=I .
$$

In the scalar case, $m_{+} \triangleq m / 2$, we obtain again Potter's equations. In the matrix case $\left(R_{i}^{\epsilon}\right)^{\frac{1}{2}}$ is needed in lower triangular form (obtained either by the Cholesky decom- position or by scalar Householder transformations) if we want to use the explicit form of $\mathcal{J}$ to compute the remaining terms $\tilde{K}_{i}, P_{i \mid i}^{\frac{1}{2}}$ in the array.

This explicit formula (29) for $\mathscr{T}$ directly yields the Andrews generalization [10a] of the Potter equations.

However we can, of course, also use these explicit formulas for our fast algorithms as well. Doing this for the constant parameter model yields the "new" equations (cf. [17])

$$
\begin{gathered}
\tilde{K}_{i}=-\tilde{K}_{i-1}\left(R_{i-1}^{\epsilon}\right)^{\frac{T}{2}}\left(R_{i}^{\epsilon}\right)^{-\frac{T}{2}} I_{ \pm}-\Phi \tilde{L_{i-1}} \tilde{L}_{i-1}^{\prime} H^{\prime}\left(R_{i}^{\epsilon}\right)^{-\frac{T}{2}} I_{ \pm} \\
\tilde{L}_{i}=\Phi \tilde{L_{i-1}}+\delta K_{i}\left[\delta\left(R_{i}^{\epsilon}\right)^{\frac{1}{2}}\right]^{-1} H \tilde{L_{i-1}}, \\
\quad\left[\left(R_{i-1}^{\epsilon}\right)^{\frac{1}{2}}, H \tilde{L}_{i-1}\right] \widetilde{T}_{0}=\left[-\left(R_{i}^{\epsilon}\right)^{\frac{1}{2}} I_{ \pm}, 0\right] \quad(30) \\
\delta K_{i}=K_{i}-K_{i-1}, \delta\left(R_{i}^{\epsilon}\right)^{\frac{1}{2}}=\left(R_{i}^{\epsilon}\right)^{\frac{1}{2}} I_{ \pm}+\left(R_{i-1}^{\epsilon}\right)^{\frac{1}{2}} .
\end{gathered}
$$

The best choice of $I_{ \pm}$remains to be investigated, as do several other variations of the above theme. However, our aim in this paper is to pursue the array method more deeply.

\section{Covariance Arrays for $C \neq 0$}

Partly as motivation for the geometric interpretations of the next section, we introduce now an extended matrix for the general state-model with $C \neq 0$. This case has several interesting features and, as we shall see, it in fact gives "true" square-root decompositions. The matrix $\mathfrak{N}$ of (10) is now replaced by the nonnegative-definite matrix

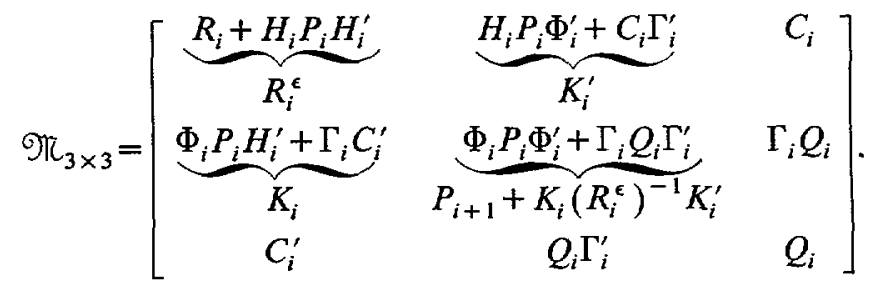

As in the previous section $\mathscr{T}_{3 \times 3}$ can be factored in two ways as $e_{1}$ and $e_{2}$.

$e_{1}$ will contain all the $a$ priori values at stage $i$ and is therefore called the "prearray."

$$
\bigodot_{1}=\left[\begin{array}{ccc}
\left(R_{i}-C_{i} Q_{i}^{-1} C_{i}^{\prime}\right)^{\frac{1}{2}} & H_{i} P_{i}^{\frac{1}{2}} & C_{i} Q_{i}^{-T / 2} \\
0 & \Phi_{i} P_{i}^{\frac{1}{2}} & \Gamma_{i} Q_{i}^{\frac{1}{2}} \\
0 & 0 & Q_{i}^{\frac{1}{2}}
\end{array}\right] .
$$

$e_{2}$ contains information related to the updated estimates (a posteriori) and is called the "post-array."

$$
\bigodot_{2}=\left[\begin{array}{ccc}
\left(R_{i}^{\epsilon}\right)^{\frac{1}{2}} & 0 & 0 \\
\tilde{K}_{i}=K_{i}\left(R_{i}^{\epsilon}\right)^{-T / 2} & P_{i+1}^{\frac{1}{2}} & 0 \\
D_{i} & E_{i} & \bar{Q}_{i}^{-\frac{1}{2}}
\end{array}\right]
$$


where $D_{i}, E_{i}, \bar{Q}_{i}^{\frac{1}{2}}$ are quantities whose actual values are not necessary for determining $\tilde{K}_{i}$ and $p^{\frac{1}{2}} i+1$. However we shall see in Section $V$ that these quantities are important in studying the so called "information-filter" form.

Note again that $e_{2}$ can be obtained from $e_{1}$ by lower triangularization using for instance Householder transformations. Now we can repeat for the general case the variations that we discussed in Section II. However we shall not go through this here. On the other hand, we feel that the strikingly simple structure of $e_{1}$ and $e_{2}$ and the fact that they are triangular calls for a more direct explanation, which we provide in the next section.

\section{GEOMETRIC INTERPRETATIONS AND DERIVATIONS}

The triangular form of the factors of $\mathfrak{T}_{3 \times 3}$ suggests that there exists an underlying Gram-Schmidt orthonormalization, which we bring out as follows.

Using the given statistics, the model equation, and the past data $y\{0, i-1\}$ we can express the random variables $\left\{y_{i}, x_{i+1}, u_{i}\right\}$ at stage $i$ in terms of certain orthonormalized variables $\left\{\mathscr{Y}_{j}, \mathcal{X}_{i}, \mathscr{Q}_{i}\right\}$ which are obtained by sequentially (from right to left) orthonormalizing, by ordinary Gram-Schmidt, the random variables $\left\{y_{i}, x_{i}, u_{i}\right\}$. We have

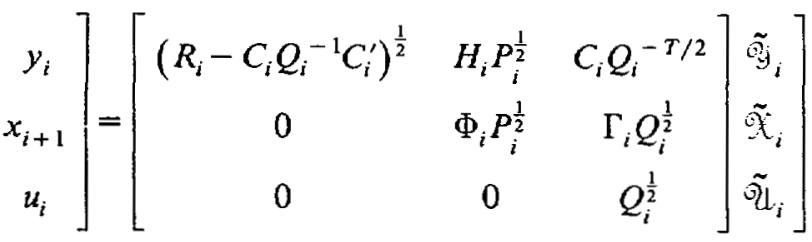

$$
\begin{aligned}
& \left.\begin{array}{c}
\hat{y}_{i \mid i-1} \\
+\hat{x}_{i+1 \mid i-1} \\
\hat{u}_{i \mid i-1}
\end{array}\right]
\end{aligned}
$$

where

$$
\begin{aligned}
& \tilde{\mathscr{Y}}_{i}=\left(R_{i}^{\tilde{y}}\right)^{-\frac{1}{2}}\left(y_{i}-\hat{y}_{i} \mid\left\{x_{i}, u_{i}, y[i-1,0]\right\}\right) \\
& \tilde{\mathscr{X}}_{i}=\left(R_{i}^{\tilde{x}}\right)^{-\frac{1}{2}}\left(x_{i}-\hat{x}_{i} \mid\left\{u_{i}, y[i-1,0]\right\}\right) \\
& \tilde{\mathcal{Q}}_{i}=\left(R_{i}^{a}\right)^{-\frac{1}{2}}\left(u_{i}-\hat{u}_{i} \mid y[i-1,0]\right)
\end{aligned}
$$

and

$$
\begin{gathered}
\hat{y}_{i} \mid x_{i}, u_{i}, y[i-1,0]=H x_{i}+C_{i} Q_{i}^{-1} u_{i} \\
\tilde{y}_{i}=y_{i}-\hat{y}_{i} \mid \cdots=v_{i}-C_{i} Q_{i}^{-1} u_{i}=\tilde{v}_{i}, \quad R_{i}^{\gamma}=R_{i}-C_{i} Q_{i}^{-1} C_{i}^{\prime} \\
\hat{x}_{i} \mid u_{i}, y[i-1,0]=\hat{x}_{i \mid i-1}, \quad R_{i}^{\bar{z}}=P_{i} \\
\hat{u}_{i} \mid y[i-1,0]=0, \quad \tilde{u}_{i}=u_{i}, \quad R_{i}^{\tilde{u}}=Q_{i}
\end{gathered}
$$

and the means are

$$
\hat{y}_{i \mid i-1}=H \hat{x}_{i \mid i-1}, \quad \hat{x}_{i+1 \mid i-1}=\Phi_{i} \hat{x}_{i \mid i-1}, \quad \hat{u}_{i \mid i-1}=0 .
$$

Note that we have obtained the prearray of (32) in the equation

$$
\left.\left.\begin{array}{c}
y_{i} \\
x_{i+1} \\
u_{i}
\end{array}\right]=e_{1}{\tilde{\tilde{X}_{i}}}_{i}\left[\begin{array}{c}
\tilde{\hat{Q}}_{i} \\
\tilde{Q}_{i}
\end{array}\right]+\begin{array}{c}
\hat{y}_{i \mid i-1} \\
\hat{x}_{i+1 \mid i-1} \\
\hat{u}_{i \mid i-1}
\end{array}\right] .
$$

Similarly, we can get the post-array by expressing the variables $\left\{y_{i}, x_{i+1}, u_{i}\right\}$ in terms of the variables $\left\{v_{i}, \tilde{X}_{i+1}, \overline{\mathscr{d}}_{i}\right\}$ obtained by orthonormalizing (from left to right) the variables $\left\{y_{i}, x_{i+1}, u_{i}\right\}$ given $y[i-1,0]$.

$$
\left.\left.\left.\begin{array}{c}
y_{i} \\
x_{i+1} \\
u_{i}
\end{array}\right]=\left[\begin{array}{cll}
\left(R_{i}^{\epsilon}\right)^{\frac{1}{2}} & 0 & 0 \\
\tilde{K}_{i} & P_{i+1}^{\frac{1}{2}} & 0 \\
D_{i} & E_{i} & \bar{Q}_{i}^{\frac{1}{2}}
\end{array}\right] \begin{array}{c}
\nu_{i} \\
\tilde{\hat{X}}_{i+1} \\
\overline{\hat{Q}}_{i}
\end{array}\right] \begin{array}{c}
\hat{y}_{i \mid i-1} \\
+\hat{x}_{i+1 \mid i-1} \\
\hat{u}_{i \mid i-1}
\end{array}\right]
$$

where

$$
\nu_{i}=\left(R_{i}^{\epsilon}\right)^{-\frac{1}{2}}\left(y_{i}-\hat{y}_{i} \mid y[i-1,0]\right)
$$

$$
=\text { the normalized innovations }
$$

$$
\begin{gathered}
R_{i}^{\epsilon}=E \epsilon_{i} \epsilon_{i}^{\prime}, \quad \epsilon_{i}=y_{i}-\hat{y}_{i \mid i-1} \\
\tilde{\hat{X}}_{i+1}=\left(R_{i+1}^{z}\right)^{-\frac{1}{2}}\left(x_{i+1}-\hat{x}_{i+1} \mid y[i, 0]\right)=P_{i+1}^{-\frac{1}{2}}\left(x_{i+1}-\hat{x}_{i+1 \mid i}\right) \\
\bar{\vartheta}_{i}=\left(R_{i}^{\bar{u}}\right)^{-\frac{1}{2}}\left(u_{i}-\hat{u}_{i} \mid x_{i+1}, y[i, 0]\right) \hat{=} \bar{Q}_{i}^{-\frac{1}{2}}\left(u_{i}-\bar{u}_{i}\right) .
\end{gathered}
$$

We may note the useful fact that $e_{1}$ and $e_{2}$ can clearly also be expressed as cross covariances,

$$
e_{1}=E\left[y_{i}^{\prime}, x_{i+1}^{\prime}, u_{i}^{\prime}\right]^{\prime}\left[\tilde{\mathscr{Y}}_{i}^{\prime}, \tilde{X}_{i}^{\prime}, \tilde{\mathscr{Q}}_{i}^{\prime}\right]
$$

and

$$
\bigodot_{2}=E\left[y_{i}^{\prime}, x_{i+1}^{\prime}, u_{i}^{\prime}\right]^{\prime}\left[v_{i}^{\prime}, \tilde{\mathcal{X}}_{i+1}^{\prime}, \overline{\text { Q }}_{i}^{\prime}\right]
$$

We may also note that we did not need the Riccati equation to obtain these arrays; as a matter of fact, the arrays could be used to derive the Riccati equation by squaring up and comparing the $(2,2)$ block elements in $e_{1} e_{1}^{\prime}$ and $e_{2} e_{2}^{\prime}$.

\section{INFORMATION FILTER}

Instead of obtaining equations for updating $P_{i}$ ( or $P_{i}^{\frac{1}{2}}$ ), it is often convenient to use $S_{i}=P_{i}^{-1}$, the inverse of the covariance matrix, especially if $P_{0}=\infty$ (no knowledge about $x_{0}$ ). Filters involving $S_{i}$ and $S_{i}^{\frac{1}{2}}$ have been called information filters. In the past, such filters have either been derived directly (see, e.g., [7], [12]) or from covariance filters by using a certain duality between the coefficients of the predicted covariance $\left(p_{i \mid i-1}\right)$ arrays and filtered information $\left(S_{i \mid i}\right)$ arrays (see, e.g., [13], [10]). Here we shall take a different route, which will actually expose a different duality from the one usually used.

We start with the prearray equation (10) and solve for the sequentially orthonormalized "prior" variables to get 
$\left[\tilde{\mathcal{Y}}_{i}, \tilde{\mathscr{X}}_{i}, \tilde{\mathcal{U}}_{i}\right]^{\prime}=\mathcal{C}_{1}^{-1}\left[y_{i}^{\prime}, x_{i+1}^{\prime}, u_{i}^{\prime}\right]^{\prime}$

$$
-\mathcal{C}_{1}^{-1}\left[\hat{y}_{i \mid i-1}^{\prime}, \hat{x}_{i+1 \mid i-1}^{\prime}, \hat{u}_{i \mid i-1}^{\prime}\right]^{\prime}
$$

where

$$
\left[\begin{array}{ll}
R_{i} & C_{i} \\
C_{i}^{\prime} & Q_{i}
\end{array}\right]>0, \quad P_{i}>0 .
$$

A similar equation can be obtained for the posterior variables $\left\{v_{i}, \tilde{\mathscr{X}}_{i+1}, \overline{\mathscr{Q}}_{i}\right\}$. Again the two sets of variables can clearly be related via an orthogonal transformation $\mathscr{T}$ (on the left). Explicit inversion of the matrices $e_{1}$ and $e_{2}$ gives the following prearray $\left[e_{1}^{-1}, \boldsymbol{m}_{1}\right]$ (where $\boldsymbol{m}_{1}$ denotes the last two columns of "mean" values):
4) We can apply this "inversion technique" to obtain information forms of all previous results. Furthermore we can obtain explicit equations either by "squaring up" the arrays or by using the explicit formulas (28) for the Householder transformation.

Squaring up gives the following equations for predicted state estimates:

$$
d_{i+1 \mid i}=\left[\Psi_{i}-L g_{i} \bar{H}_{i}\right] d_{i \mid i-1}+\left[\bar{\Gamma}_{i} \bar{R}_{i}^{-1}-L g_{i} \bar{C}_{i}\right] y_{i}
$$

where

$$
d_{i+1 \mid i} \triangleq P_{i+1}^{-1} \hat{x}_{i+1 \mid i}, L g_{i} \triangleq \bar{L}_{i} \bar{M}_{i}^{-1}
$$

\begin{tabular}{c|cc|c|c|c}
\hline & $y_{i}$ & $x_{i+1}$ & $u_{i}$ & $1 \mid y[i-1,0]$ & $1 \mid y[i, 0]$ \\
\hline$\tilde{\mathcal{Y}}_{i}$ & $\tilde{V}_{i}$ & $-\bar{R}_{i}^{-\frac{1}{2}} H_{i} \Phi^{-1}$ & $C_{i}$ & 0 & $\bar{R}_{i}^{-\frac{1}{2}} y_{i}$ \\
$\tilde{X}_{i}$ & 0 & $P_{i}^{-\frac{1}{2}} \Phi_{i}^{-1}$ & $-P_{i}^{-\frac{1}{2}} \Phi_{i}^{-1} \Gamma_{i}$ & $P_{i}^{-\frac{1}{2}} \hat{x}_{i \mid i-1}$ & $-P_{i \mid i}^{-\frac{1}{2}} \hat{x}_{i \mid i-1}$ \\
$\tilde{\vartheta}_{i}$ & 0 & 0 & $Q_{i}^{-\frac{1}{2}}$ & 0 & 0 \\
\hline
\end{tabular}

where

$$
\begin{gathered}
\tilde{V}_{i}=\left(\bar{R}_{i}\right)^{-\frac{1}{2}} \triangleq\left(R_{i}-C_{i} Q_{i}^{-1} C_{i}^{\prime}\right)^{-\frac{1}{2}}, \\
\tilde{C}_{i} \triangleq\left(\bar{R}_{i}\right)^{-\frac{1}{2}}\left[H_{i} \Phi_{i}^{-1} \Gamma_{i}-C_{i} Q_{i}^{-1}\right]
\end{gathered}
$$

and the following duality table can be obtained by using inversion

$$
\left[\begin{array}{c}
\Phi_{i} \leftrightarrow \Psi_{i} \triangleq \Phi_{i}^{-T} \\
H_{i} \leftrightarrow \bar{H}_{i} \triangleq \Gamma_{i}^{\prime} \Psi_{i} \\
\Gamma_{i} \leftrightarrow \bar{\Gamma}_{i} \triangleq \Psi_{i} H_{i}^{\prime}
\end{array}\right]
$$

and the following post-array $\left[e_{2}^{-1}, m_{2}\right]$

\begin{tabular}{c|cc|cc|c}
\hline & $y_{i}$ & $x_{i+1}$ & $u_{i}$ & $1 \mid y[i-1,0]$ & $1 \mid y[i, 0]$ \\
\hline$v_{i}$ & $\left(R_{i}^{\epsilon}\right)^{-\frac{1}{2}}$ & 0 & 0 & $-\left(R_{i}^{\epsilon}\right)^{-\frac{1}{2}} H_{i} \hat{x}_{i \mid i-1}$ & $p_{i}$ \\
$\tilde{X}_{i+1}$ & $-P^{-\frac{1}{2}} K g_{i}$ & $P^{-\frac{1}{2}}{ }_{i+1}$ & 0 & $-P_{i+1}^{-\frac{1}{2}} \Phi_{c i} \hat{x}_{i \mid i-1}$ & $-P_{i+1}^{-\frac{1}{2}} \hat{x}_{i+1 \mid i}$ \\
$\vec{Q}_{i}$ & $\bar{D}_{i}$ & $-\bar{Q}_{i}^{-\frac{1}{2}} E_{i} P_{i+1}^{-\frac{1}{2}}$ & $\bar{Q}_{i}^{-\frac{1}{2}}$ & $-\bar{Q}_{i}^{-\frac{1}{2}} \hat{u}_{i \mid i-1}$ & $-\bar{Q}_{i}^{-\frac{1}{2}} \hat{u}_{i \mid i}$ \\
\hline
\end{tabular}

where

$$
\begin{aligned}
& \bar{D}_{i} \triangleq-\left(\bar{Q}_{i}\right)^{-\frac{1}{2}}\left[E_{i} P^{-\frac{1}{2}}{ }_{i+1} K g_{i}-D_{i}\left(R_{i}^{\epsilon}\right)^{-\frac{1}{2}}\right] \\
& \Phi_{c i} \triangleq\left(\Phi-K g_{i} H_{i}\right), \quad K g_{i} \triangleq K_{i}\left(R_{i}^{\epsilon}\right)^{-1} .
\end{aligned}
$$

\section{Remark:}

1) It is customary to leave the state estimates $P_{i}^{-\frac{1}{2}} \hat{x}_{i \mid i-1}$ "normalized" if not explicitly needed.

2) Note that the estimates $\hat{x}_{i \mid i-1}$ and $\hat{x}_{i+1 \mid i}$ need not be separately calculated as in the covariance forms. Here we can transform the means " $1 \mid y[i, 0]$ " directly by $\mathscr{T}$, thus, the $y_{i}$ block column is not needed.

3) The $3 \times 3$ matrix provides us with a means of switching between the covariance and information forms, (even of combining them if some states are completely unknown $[P]_{i j}=\infty$ and some are completely known $[P]_{j j}$ $=0$ ).

$$
\left[\begin{array}{c}
P_{i+1} \leftrightarrow S_{i+1} \triangleq P_{i+1}^{-1} \\
K_{i} \leftrightarrow \bar{L}_{i} \triangleq \Psi_{i} S_{i} \bar{H}_{i}^{\prime}+\bar{\Gamma}_{i} \bar{C}_{i}^{\prime} \\
R_{i}^{\epsilon} \leftrightarrow \bar{M}_{i} \triangleq \bar{H}_{i} S_{i} \bar{H}_{i}^{\prime}+\bar{Q}_{i}^{-1}
\end{array}\right]
$$

$$
\left[\begin{array}{l}
Q_{i} \leftrightarrow \bar{R}_{i}^{-1} \triangleq\left(R_{i}-C_{i} Q_{i} C_{i}^{\prime}\right)^{-1} \\
C_{i} \leftrightarrow \bar{C}_{i}^{\prime} \triangleq \bar{R}_{i}^{-1}\left(H_{i} \Phi_{i}^{-1} \Gamma_{i}-C_{i} Q_{i}^{-1}\right) \\
R_{i} \leftrightarrow \bar{Q}_{i}^{-1} \triangleq Q_{i}^{-1}+\bar{C}_{i} \bar{R}_{i} \bar{C}_{i}^{\prime}
\end{array}\right] .
$$

5) The above duality relations are different from those usually used (see, e.g., [9], [10]), which we may call the "standard" duality. We may note however that the standard duality gets rather complicated when $C_{i} \neq 0$ and no formulas for this case have been explicitly given in the literature. Clearly also we now have several possible ways 
of relating predicted and filtered covariance and information quantities, as diagrammed in Fig. 1.

\section{Given Covariance Information}

An alternative solution to the filtering problem can be given if instead of a state-space model, we only have covariance information in the form

$$
\begin{aligned}
& R_{i j}^{y}=H_{i} \Phi_{i}^{i-j} N_{j}, \quad i>j \\
& R_{j i}^{y}=R_{i j}^{y \prime} .
\end{aligned}
$$

If there is an underlying state-space model of the form (1), we can identify

$$
N_{i}=\Pi_{i} H_{i}^{\prime}+\Gamma_{i} C_{i}^{\prime}, \quad \Pi_{i} \hat{=} E x_{i} x_{i}^{\prime} .
$$

The Riccati equation solution is given by [26]

$$
\begin{gathered}
\hat{x}_{i+1 \mid i}=\Phi_{i} \hat{x}_{i \mid i-1}+\tilde{K}_{i} \nu_{i} \\
\nu_{i}=\left(R_{i}^{\epsilon}\right)^{-\frac{1}{2}} \epsilon_{i}, \quad \epsilon_{i}=y_{i}-H_{i} \hat{x}_{i \mid i-1} \\
R_{i}^{\epsilon}=R_{i i}^{y}-H_{i} \Sigma_{i} H_{i}^{\prime}, \quad \tilde{K}_{i}=\Phi_{i}\left(N_{i}-\Sigma_{i} H_{i}^{\prime}\right)\left(R_{i}^{\epsilon}\right)^{-\frac{T}{2}} \\
\Sigma_{i+1}=\Phi_{i} \Sigma_{i} \Phi_{i}+\tilde{K}_{i} \tilde{K}_{i}^{\prime}, \quad \Sigma_{0}=0 .
\end{gathered}
$$

However, we can also develop square-root algorithms. Let $\Re_{c}=\left[\begin{array}{ll}R_{i i}^{y}-H_{i} \Sigma_{i} H_{i}^{\prime}=R_{i} & \left(N_{i}^{\prime}-H_{i} \Sigma_{i}\right) \Phi_{i}^{\prime}=K_{i}^{\prime} \\ -\Phi_{i}\left(N_{i}-\Sigma_{i} H_{i}^{\prime}\right)=K_{i 1}-\Phi_{i} \Sigma_{i} \Phi_{i}^{\prime}=K_{i}\left(R_{i}^{\mathrm{c}}\right)^{-1} K_{i}^{\prime}-\Sigma_{i+1}\end{array}\right]$.

Note that our previous

$$
\Re=\Re_{c}+\left[\begin{array}{ll}
0 & 0 \\
0 & \Pi_{i+1}
\end{array}\right] .
$$

Again we can factor $\Re_{c}$ into $e_{1} e_{1}^{\prime}$ and $e_{2} e_{2}$, where

$$
\Theta_{1}=\left[\begin{array}{lll}
R_{i i}^{y^{\frac{1}{2}}} & \sqrt{-1} H_{i} \Sigma_{i}^{\frac{1}{2}} & 0 \\
\Phi_{i} N_{i} R_{i i}^{y^{-\frac{T}{2}}} & \sqrt{-1} \Phi_{i} \Sigma_{i}^{\frac{1}{2}} & \sqrt{-1} \Phi_{i} N_{i} R_{i i}^{y^{-\frac{T}{2}}}
\end{array}\right]
$$

and

$$
\varrho_{2}=\left[\begin{array}{lll}
R_{i}^{\epsilon^{\frac{1}{2}}} & 0 & 0 \\
K_{i}\left(R_{i}^{\epsilon}\right) & \sqrt{-1} \sum_{i+1}^{\frac{1}{2}} & 0
\end{array}\right]=\varrho_{1} \widetilde{\top}
$$

However, we now have arrays with imaginary columns, but no complex arithmetic will be needed in the computation. We need only to keep track of "purely imaginary" and "purely real" columns [10, p. 77].

If $\Phi$ and $H$ are constant we can develop fast algorithms.

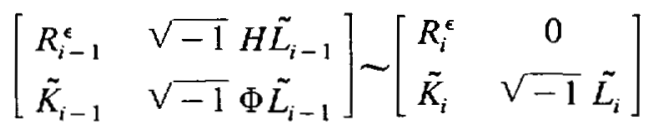

where

$$
\delta \Sigma_{i}=\Sigma_{i+1}-\Sigma_{i}=\tilde{L}_{i} \tilde{L}_{i}^{\prime} .
$$

The proper initial values for these arrays needs a separate

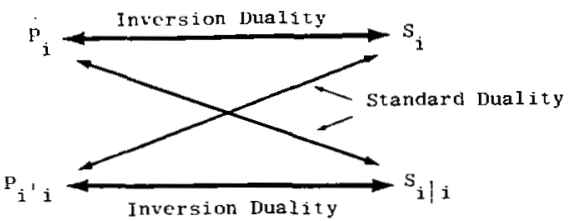

Fig. 1.

calculation described in [14]. However in the stationary case, where $N_{i}=N, R_{i i}^{y}=R_{0}^{y}=R_{0}^{\epsilon}$, they are easily specified as

$$
\tilde{K}_{0}=\tilde{L}_{0}=\Phi N\left(R_{0}^{y}\right)^{-\frac{T}{2}} .
$$

\section{Concluding Remarks}

For reasons of space, discussions of several extensions have been postponed to a later paper. However, in the Appendices, brief discussions are given of some of these. Appendix A briefly discusses smoothing problems, Appendix $B$ notes possible extensions to time-variant systems, and Appendix $\mathrm{C}$ discusses continuous systems, especially those with discrete measurements. This last problem is capable of quite efficient numerical solutions as studied in some detail by Potter and Womble [25]; our presentation is made chiefly for completeness.

There are also relations to nonstochastic problems. Needless to say, by the well known duality [27], our results can be applied to the quadratic regulator problem. We are also studying array methods for minimal realization and identification. In his dissertation, Dickinson [24] has pursued the application of our fast algorithms to the explicit calculation of the various (controllability and observability) subspaces that arise in the geometric theories of control of Wonham, Morse, et al.

This last application was stimulated by a paper of Payne and Silverman [23]. This paper contains a development via a dynamic programming argument of certain square-root algorithms for the Riccati equation of quadratic control. In the time-invariant case, they obtain arrays dual to those in our equation (24). They also established some connections with our explicit equations in [14].

We are happy to acknowledge that [23] along with the work of Golub [5] and Kaminski [10] were important stimuli for our work.

\section{APPENDIX A}

\section{Arrays for Smoothing}

There exists a host of smoothing equations and our method seems to be powerful enough to rederive all of them as well as some new ones. The choice of the proper variables and the many possibilities of ordering them (to get the orthonormal coordinate systems) increases considerably for the smoothing problem. The choice of useful variables for the smoothing equations has been more of 
an art than a science. We feel that the array-methods provide a more systematic approach to this choice (for instance by enumerating all possible ones), thereby also filling some gaps in earlier studies which worked with only some of the possible choices. A detailed study of smoothing problems will be deferred to a later paper but as an example of our results, we give a new array method for the fixed-point smoothing problem, where we have to estimate $x_{k}$ given $\left\{y[k, k+1, \cdots, i], \hat{x}_{k \mid k-1}\right\}$. The prearray equation is

$$
\left.\begin{array}{c}
y_{i} \\
x_{i+1} \\
x_{k} \\
u_{i}
\end{array}\right]=\left[\begin{array}{ccc}
\left(R_{i}-C_{i} Q_{i}^{-1} C_{i}^{\prime}\right)^{\frac{1}{2}} & H_{i} P_{i \mid x_{k}}^{\frac{1}{2}} & H_{i} R_{i, k}^{x} P_{k \mid i-1}^{\frac{1}{2}} \\
0 & \Phi_{i} P_{i \mid x_{k}}^{\frac{1}{2}} & \Phi_{i} R_{i, k}^{x} P_{k \mid i-1}^{\frac{1}{2}} \\
0 & 0 & P_{k \mid i-1}^{\frac{1}{2}} \\
0 & 0 & 0
\end{array}\right.
$$

with initial conditions

$$
P_{k \mid x_{k}}=0, \quad R_{k, k}^{x} \hat{=} I .
$$

Note that all the quantities $\left\{\tilde{\mathscr{Y}}_{i}, \tilde{X}_{i}, \tilde{\mathscr{Q}}_{i}\right\}$ are conditioned or "pinned" on $x_{k}$, a feature that arises naturally in the $G-S$ procedure applied here.

By a further modification of the array in (Al) (i.e., by a second "pass" over the data) we could derive for instance the smoothing formula of Kaminski and Bryson [9], [10]. However, a somewhat simpler (one-pass) procedure can be given by triangularizing the prearray to get

\begin{tabular}{c|cc|cc|c}
\hline & $\nu_{i}$ & $\tilde{X}_{k \mid i}$ & $\tilde{X}_{i+1 \mid i, x_{k}}$ & $\bar{Q}_{i \mid i, x_{k}, x_{i}}$ & $1 \mid i-1$ \\
\hline$y_{i}$ & $\left(R_{i}\right)^{\frac{1}{2}}$ & 0 & 0 & 0 & $H_{i} \hat{x}_{i \mid i-1}$ \\
$x_{k}$ & $\tilde{K}_{k \mid i}$ & $P_{k \mid i}^{\frac{1}{2}}$ & 0 & 0 & $\hat{x}_{k \mid i-1}$ \\
$x_{i+1}$ & $\tilde{K}_{i}$ & $R_{i+1, k}^{x} P_{k \mid i}^{\frac{1}{2}}$ & $P_{i+1 \mid x_{k}}^{\frac{1}{2}}$ & 0 & $\Phi \tilde{x}_{i \mid i-1}$ \\
$u_{i}$ & $D_{i}$ & $L_{i, k}$ & $L_{i \mid x_{k}}$ & $\bar{Q}_{i}^{\frac{1}{2}}$ & 0 \\
\hline
\end{tabular}

Remark: Notice that in effect we could augment the state $x_{i}$ by $x_{k}$ to get the above results from the filtering arrays (32), (33), an idea apparently first used by Zachrisson [21].

\section{APPENDIX B}

Generalization of the Fast Forms to Time Variant $R_{i}$ and $Q_{i}$

The crucial facts for obtaining the fast algorithm of Section II was the constancy of $H$ and $\Phi$. Time variations of $R_{i}$ and $\Gamma_{i}, Q_{i}$ can be handled just as for $P_{i}$. Namely, suppose again that

$$
R_{i+1}=R_{i}+\delta R_{i} \quad \text { and } \quad \delta R_{i}=V_{i} V_{i}^{\prime}
$$

$$
\Gamma_{i+1} Q_{i+1} \Gamma_{i+1}=\Gamma_{i} Q_{i} \Gamma_{i}^{\prime}+\delta \theta_{i} \quad \text { and } \quad \delta \theta_{i}=U_{i} U_{i}^{\prime}
$$

(where $V_{i}$ and $U_{i}$ might have imaginary columns). Thus, we will have

$$
R_{i+1}^{\frac{1}{2}} \sim\left[R_{i}^{\frac{1}{2}}, V_{i}\right], \Gamma_{i+1} Q_{i}^{\frac{1}{2}} \sim\left[\Gamma_{i} Q_{i}^{\frac{1}{2}}, U_{i}\right] .
$$

We remark at this point that this expansion should not be regarded as a trick, but rather as a kind of first-order Taylor series expansion of the variables of the fast-array

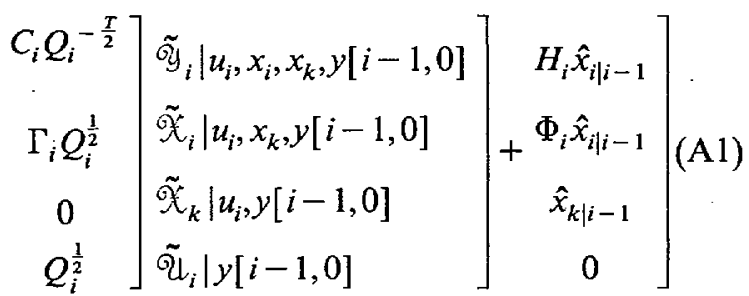

or terms of variables of the prearray, noting that we then can disregard the "constant term" of the series, because it appears in both arrays.

With the use of the expansion (B3) we can now expand the prearray (16) as

$$
\begin{gathered}
p \\
n\left[\begin{array}{ccccccc}
p & p & n & \alpha_{i-1} & m & m \\
R_{i-1}^{\frac{1}{2}} & V_{i-1} & H P_{i-1}^{\frac{1}{2}} & H \tilde{L}_{i-1} & 0 & 0 \\
0 & 0 & \Phi P_{i-1}^{\frac{1}{2}} & \Phi \tilde{L}_{i-1} & \Gamma_{i-1} Q_{i-1}^{\frac{1}{2}} & U_{i-1}
\end{array}\right] \\
\\
\sim\left[\begin{array}{cccccc}
\left(R_{i-1}^{\epsilon}\right)^{\frac{1}{2}} & 0 & 0 & V_{i-1} & H \tilde{L}_{i-1} & 0 \\
\tilde{K}_{i-1} & P_{i}^{\frac{1}{2}} & 0 & 0 & \Phi \tilde{L}_{i-1} & U_{i-1}
\end{array}\right] \\
\sim \\
\quad n\left[\begin{array}{cccc}
p & n & \delta_{i} & \alpha_{i} \\
\left(R_{i}^{\epsilon}\right)^{\frac{1}{2}} & 0 & 0 & 0 \\
\tilde{K}_{i} & P_{i}^{\frac{1}{2}} & 0 & \tilde{L}_{i}
\end{array}\right]
\end{gathered}
$$

where $\alpha_{i}$ is a number such that

$$
\alpha_{i} \leqslant p+m+\alpha_{i-1}=\delta_{i}+\alpha_{i}, \quad \alpha_{j} \leqslant n .
$$

Unlike the case of constant $R_{i}$ and $Q_{i}$ there the rank of $\tilde{L}_{i}$ can increase or decrease with $i$ and unless we wish to particularly exploit this fact, we will have to reserve $n$ columns for the $\left\{\tilde{L}_{i}\right\}$, so that, in general, we would need at most $p$ wave columns than for (16). However, in some applications $\alpha_{i}$ might be less than $n-p$ for all $i$; for instance, if the system has more than $p$ degenerate directions [19].

\section{General Time Variant Case}

We note briefly that the requirements of constant $H$ and $\Phi$ can be circumvented by the artifice of reducing the 
state equation for a time varying system $\Sigma\left(H_{i}, \Phi_{i}, \Gamma_{i}\right) \cdot$ to a time varying autoregressive moving average (ARMA) model of the form

$$
y_{i}+A_{1, y_{i-1}}+\cdots+A_{n, i} y_{i-n}=w_{i}, \quad i \geqslant n, A_{0, i}=I
$$

where $w_{i}$ is a finitely-correlated MA process

$$
w_{i}=B_{1, i} u_{i-1}+\cdots+B_{n, i} u_{i-n}, \quad i \geqslant n, B_{0, i}=0 .
$$

The reduction to this model (see [17]) corresponds to factoring the impulse response matrix of $\Sigma\left(H_{i}, \Phi_{i}, \Gamma_{i}\right)$

$$
[\varrho]_{i j}=L_{i j}= \begin{cases}H_{i} \Phi_{i-1} \cdots \Phi_{j+1} \Gamma_{j}, & i>j+1 \\ H_{i} \Gamma_{j}, & i=j+1 \\ 0, & \text { otherwise }\end{cases}
$$

as

$$
\mathfrak{L}=\mathbb{Q}^{-1} \mathscr{G},[\mathbb{Q}]_{i j}=A_{j-i, i},[\mathscr{B}]_{i j}=B_{j-i, i}
$$

where $A \cdot, .=0$ and $B \cdot, \cdot=0$ for $j-i \notin[0, n]$. Now it can be arranged [16] that the innovations of $\left\{y_{i}\right\}$ are equal to those of $\left\{w_{i}\right\}$, so that we need only concern ourselves with the $\left\{w_{i}\right\}$ processes. But since $\left\{w_{i}\right\}$ is an MA process it is easy to see that it can be represented in the state-space form () with

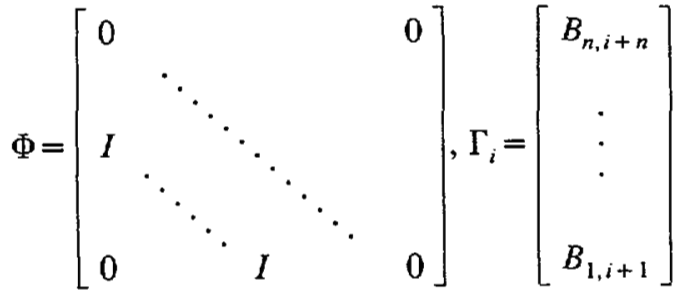

$$
\begin{aligned}
& H=[0 \cdots I], \quad R=0, \quad Q_{i}=I \\
& w_{i}=H x_{i}, \quad x_{i+1}=\Phi x_{i}+\Gamma_{i} u_{i} \text {. }
\end{aligned}
$$

But now $H, \Phi$ are constant so that the previous arguments can be applied. We note that this constancy yields a "shift-invariance" property, which is exploited differently in [15] to get an innovations derivation of the Chandrasekhar-type equations (see also [17], [20]).

\section{APpendix C}

\section{Square Root Techniques for Continuous-Time Systems}

There do not seem to be natural analogs in continuous time of the previous equations. To give an example of what is possible, note that if we define

$$
P(t)=S(t) S^{\prime}(t)
$$

and assume $P(t)>0$, then from the continuous-time Riccati equation

$$
\begin{aligned}
& \dot{P}(t)=F(t) P(t)+P(t) F^{\prime}(t)-K(t) K^{\prime}(t)+Q(t) \\
& K(t)=P(t) H^{\prime}(t)+C(t) .
\end{aligned}
$$

Andrews [10a] obtained with $A+A^{\prime}=0$ the equation

$$
\dot{S}=\left[F-\left(C+\frac{1}{2} S S^{\prime} H^{\prime}\right) H\right] S+\left[A+\frac{1}{2}\left(Q-C C^{\prime}\right)\right]\left(S^{-1}\right)^{\prime}
$$

This is not very convenient because of the need to use a similar equation for $S^{-1}$. However, the antisymmetric matrix $A$ can be chosen such that $\dot{S}$ and $S$ is triangular [10a], so that $S^{-1}$ is easily computed. We may note that $S$ obeys a nicer equation when $Q-C C^{\prime}=0$ (i.e., there is no plant noise).

Another variation that does not require $P(t)>0$ but assumes knowledge of one solution of the Riccati equation [e.g., of the algebraic Riccati equation (for constant systems)] is the following.

Let $P_{1}(t), P_{2}(t)$ be two solutions of the Riccati equation. Then we can show that

$$
\begin{gathered}
P_{1}(t)=P_{2}(t)+S(t) S^{\prime}(t) \\
\dot{S}=\left[F-\left[\left(P_{2}+\frac{1}{2} S S^{\prime}\right) H^{\prime}+C\right] H\right] S, \\
S(0)=\left[P_{1}(0)-P_{2}(0)\right]^{\frac{1}{2}} .
\end{gathered}
$$

More use can be made of square-root ideas if the continuous-time Riccati equation is replaced by a particular discretized equivalent, as recently proposed by Potter and Womble [25]. They noted that the solution of the continuous-time Riccati equation at discrete times $0=t_{0}<t_{1}<t_{2} \cdots$ could be computed via recursions that constant systems and uniform sampling (i.e., $t_{k+1}-t_{k}=\Delta$ ), have the form

$$
P_{k+1}=P_{*}+A\left[W+P_{k}^{-1}\right]^{-1} A^{\prime}
$$

where $P_{*}, A, W$ are quantities obtained by integration over $\Delta$ of a Riccati equation with zero initial conditions. As Potter and Womble note, these equations are in a form to which discrete-time square-root ideas can be readily applied. (This possibility was also noted by Bierman, personal communication.) Thus let

$$
P_{k}=S_{k} S_{k}^{\prime}
$$

Then (C4) can be written

$$
S_{k+1} S_{k+1}^{\prime}=P^{\frac{1}{2}} P^{\frac{T}{2}}+A S_{k}\left(M_{k}^{\prime} M_{k}\right)^{-1} S_{k}^{\prime} A^{\prime}
$$

where

$$
M_{k}^{\prime} M_{k}=I+S_{k}^{\prime} W^{\frac{1}{2}} W^{\frac{T}{2}} S_{k}
$$

Now it is obvious that

$$
\begin{aligned}
S_{k+1} & \sim\left[P^{\frac{1}{2}}{ }_{*}, A S_{k} M_{k}^{-1}\right] \\
M_{k} & \sim\left[I, W^{\frac{T}{2}} S_{k}\right] .
\end{aligned}
$$

Note that we can take $M_{k}$ to be a triangular square-root so that its inverse can be more easily computed. 


\section{REFERENCES}

[1] R. E. Kalman, "A new approach to linear filtering and prediction problems," Trans. ASME, (J. Basic Eng.), vol. 82D, pp. $34-45$, Mar. 1960.

[2] J. H. Wilkinson and C. Reinsch, Linear Algebra, Handbook of Automatic Computation, vol. 2. Berlin: Springer, 1971 .

[3] V. Kucera, "A review of the discrete-time matrix Riccati equation," Kybernetica, Vol. 8, pp. 430-447, 1972.

[4] J. E. Potter and R. G. Stern, "Statistical filtering of space navigation measurements," Proc. 1963 AIAA Guidance and Control Conf. 1963.

[4a] R. H. Battin, Astronautical Guidance. New York: McGraw-Hill, 1964, pp. 338-340.

[5] G. H. Golub, "Numerical methods for solving linear least squares problems," Numer. Math., vol. 7, pp. 206-216, 1965.

[6] R. J. Hanson and C. L. Lawson, "Extensions and applications of the Householder algorithm for solving linear least-squares problems," Math. Comput. vol. 23, pp. 787-812, 1969

[7] P. Dyer and S. McReynolds, "Extension of square-root filtering to include process noise," J. Optimiz. Theory $A p p l$., vol. 3, pp. 444 $459,1969$.

[8] S. F. Schmidt, "Computational techniques in Kalman filtering," Nato-AGARD-139, Feb. 1970

[9] P. G. Kaminski and A. E. Bryson, "Discrete square root smoothing," Proc. AIAA Guidance and Control Conf., 1972, paper 72-877.

[10] P. G. Kaminski, "Square root filtering and smoothing for discrete processes," Ph.D. dissertation, Dep. Aerosp. Astronaut., Stanford Univ., Stanford, Calif., Sept. 1971 .

[10a] A. Andrews, "A square root formulation of the Kalman covariance equations," AIAA J., vol. 6, pp. 1165-1166, June 1968.

1) G. J. Bierman, "A comparison of discrete linear filtering algorithms," IEEE Trans. Aerosp. Electron. Syst., vol. AES-9, pp. 28-37, Jan. 1973.

[12] - "Sequential square root filtering and smoothing of discrete linear systems," Automatica, vol. 10, pp. 147-158, Mar. 1974.

[13] T. Kailath, "Some new algorithms for recursive estimation in constant linear systems," IEEE Trans. Inform. Theory, vol. IT-19, pp. $750-760$, Nov. 1973

[14] M. Morf, G. S. Sidhu, and T. Kailath, "Some new algorithms for recursive estimation in constant, linear, discrete-time systems," IEEE Trans. Automat. Contr., vol. AC-19, pp. 315-323, Aug. 1974, also pres.

[15] G. S. Sidhu, T. Kailath, and M. Morf, "Development of fast algorithms via innovations decomposition." in Proc. of the 7 th Hawaii Int. Conf. Systems Sciences, Honolulu, Hawaii, Jan. 1974, pp. 192-195.

[16] B. W. Dickinson, T. Kailath, and M. Morf, "Canonical matrix B. W. Dickinson, T. Kailath, and M. Morf, "Canonical matrix
fraction and state-space description for deterministic and fraction and state-space description for deterministic and stochastic linear systems," IEEE Trans. Automat. Contr. (Special
Issue on System Identification and Time-Series Analysis), vol. AC-19, pp. 656-667, Dec. 1974 .

[17] M. Morf, "Fast algorithms for multivariable systems," Ph.D. dis(M)

18] D. Rappaport, "Constant directions of the Riccati equation," Automatica, vol. 8, pp. 175-186, Mar. 1972.

R. S. Bucy, D. Rappaport, and L. M. Silverman, "Correlated noise filtering and invariant directions for the Riccati equation," IEEE filtering and invariant directions for the Riccati equation,"
Trans. Automat. Contr., vol. AC-15, pp. 535-540, Oct. 1970.

[19] M. Gevers and T. Kailath, "Constant, predictable and degenerate directions of the discrete-time Riccati equation," Automatica, vol. 9 pp. $699-711,1973$.

[20] G. S. Sidhu and T. Kailath, "Development of new estimation algorithms by innovations analysis and shift-invariance properties," IEEE Trans. Inform. Theory, vol. IT-20, pp. 759-762, Nov.

1] L. Zachrisson, "On optimal smoothing of continuous-time Kalman L. Zachrisson, "On optimal smoothing of continuo

[22] M. Morf and T. Kailath, "Square-root algorithms for least-squares estimation and control," in Proc. 8th Princeton Symp. Information and System Sciences Princeton, N.J., Mar. 1974.

[23] H. J. Payne and L. M. Silverman, "Matrix Riccati equations and systems structure," in 1973 IEEE Decision and Control Conf., San Diego, Calif., Dec. 1973.

[24] B. Dickinson, "Properties and applications of matrix fraction descriptions of linear systems," Ph.D. dissertation, Dep. Elec. Eng., Stanford Univ., Stanford, Calif., 1974

[25] M. E. Womble and J. E. Potter, "A prefiltering version of the Kalman filter with new numerical integration formulas for Riccati equations," in Proc. 1973 IEEE Decision and Control Conf., San Diego, Calif., pp. 63-67.

[26] M. R. Gevers and T. Kailath, "An innovations approach to least-squares estimation-Part VI: Discrete-time innovations representations and recursive estimation," IEEE Trans. Automai. Contr., vol. AC-18, pp. 588-600, Dec. 1973.
[27] H. Kwakernaak and R. Sivan, Linear Optimal Control Systems. New York: Wiley, 1972.

[28] J. R. Bunch and B. N. Parlett, "Direct method for solving symmetric indefinite systems of linear equations," SIAM J. Numer. Anal., vol. 8, pp. 639-655, 1971

[29] T. Kailath, B. Dickinson, M. Morf, and G. S. Sidhu, "Some new algorithms for recursive linear estimation and related problems," in Proc. 1973 IEEE Decision and Control Conf., San Diego, Calif., Dec. 1973 , pp. 556-557.

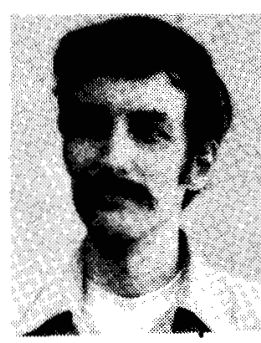

Martin Morf (S'70-M'74) was born in Winerthur, Switzerland, on June 16,1944 . He received the Engineer's degree in electrical enginology, Zuich, Swilzerland in 1968, the M.S.E. E. degree and the Ph.D. degree from Stanford E. degree and the Ph.D. degree from Stanford
University, Stanford, Calif., in 1970 and 1974, respectively.

In 1968 he was a Research Assistant at the Technical Physics Laboratory, Federal Institute of Technology, Zürich, and from 1969 to 1972 at Stanford University In 1973 he was a Research Associate and since 1974 he has been acting as Assistant Professor in the Information Systems Laboratory at Stanford. His research interests are in information theory, the areas of structure of multivariable systems, estimation, identification, control, and efficient computations. He is also interested in applications of these areas to speech processing and large scale systems.

Dr. Morf is a member of Sigma Xi.

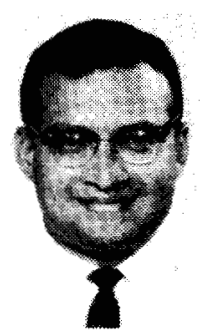

Thomas Kailath (S'57-M'62-F'70) was born in Poona India, on June 7,1935 . He received the B.E degree in telecommunications engineering from the Universy of Poona, Poona, India, in 1956 and the S.M. and Sc.D. degrees in electriof Technology, Cambridge, in 1959 and 1961, respectively.

During 1961-1962 he worked at the Jet Propulsion Laboratories, Pasadena, Calif. He also taught part time at the California Institute of Technology, Pasadena. From 1963 to 1968, he was Associate Professor, and from 1968 Professor, of Electrical Engineering at Stanford University, Stanford, Calif., where he is now Director of the Information Systems Laboratory. During January-June 1963, he was a Visiting Scholar at the University of California, Berkeley. From 1969 to 1970 he held a Guggenheim Fellowship and was also a Visiting Professor at the Indian Institute of Science, Bangalore. He has also spent summers at the MIT Lincoln Laboratories, Bell Laboratories, Imperial College, and the Indian Statistical Institute, and has consulted for several industrial companies. His research interests are generally in statistical signal processing, especially in communications and control. With a co-author he won the 1965-1966 outstanding paper prize of the IEEE Information Theory Group. He was Guest Editor in 1974 of a Special Issue on System Identification and Time-Series Analysis of the IEEE Transactions on Automatic Control. He is Editor of a Prentice-Hall series of books on information and system sciences.

Dr. Kailath is a member of the Editorial Board of the IEEE Press. From 1971 to 1974 he was Associate Editor for Detection and Estimation Theory of the IEEE Transactions on Information Theory. Since 1971, he has been a member of the Administrative Committees of the IEEE Professional Group on Information Theory and of the IEEE Control Systems Society, and during 1975 he is serving as President of the IEEE Information Theory Group. $\mathrm{He}$ is a member of the Institute of Mathematical Statistics, Commission VI of URSI (the International Scientific Radio Union), Sigma Xi, and several other scientific societies. 